Mind as Selective Process System

Raimo J Laasonen

Nummela

Finland 


\begin{abstract}
First, the objective of the current research was to widen the model of the mind dynamic. Second, the objective was to develop elaboration because its development appeared to have remained incomplete, especially with probabilities. Three processes were definable: Selector, Opter, and Chooser in the mind. It was hypothesized that gender, socioeconomic status, occupational class, and social environment regulate action of Selector, Opter, and Chooser. The number of the participants was 186, 130 males, and 56 females. There were 34 selfemployed persons; 47 upper level employees; 83 lower level employees, and 22 manual workers. The distribution of the occupational class was 68 leaders, 62 experts, 16 officers, and 40 servants. The causal analysis the processes comprised of elaboration, and of utilizing matrix calculus with probabilities. The hypothesis corroborated, and the results indicated that the causation included in one to one, many to one, and one to many causal mappings. KEY WORDS: Selector, Opter, Chooser, elaboration, percept, matrix power
\end{abstract}




\section{Mind as Selective Process System}

First, the objective of the current research was to widen the model of the mind dynamic (Laasonen, 2007, p.134). Shortly, the model attempted to tackle mind processes that may be essential to the mind as a process system where processes process processes. Second, the objective was to develop elaboration because its development appeared to have remained incomplete, especially with probabilities. In a methodical sense, elaboration means introducing a third variable into a two-variable relationship, and to examine whether the original relationship changes because of variable elimination. The framework of the former mind researches has been systems analysis, dynamically.

Since Bertalanffy (1968) debate has continued about systems, their nature, and realization. Presently, direction seems to be towards dynamic in the systems, for example, Freyd (1987, pp.1-12), Fusella (2013, pp.1-14). Dynamic presupposes existence of processes, and in the mind researches transition to processes is weakly in sight. The former representations replace with processes Bickhard (2002, pp.1-15). There have been discussions about the mind as a calculator Piccini and Scarantino (2010, pp. 1-38). Well, if mind is a calculator, why then is it difficult to learn mathematics? Maybe mind is a calculator but the guess is, it has mathematics of its own. Constructions have emerged, which deal with architecture of the mind but in a systemic sense, question is about structure, plainly. Modularity of the mind has also received foot step in researching the mind dynamic but modularity includes in replacement, implicitly Prinz (2006, pp.1-15). Lately, dynamical systems theory has become in daylight, and it has been applied to complete shortcomings of process approach. One thing that has not sprung up often is the question of causal process systems in the mind. The current research tackles the problem. Therefore, it was necessary to 
construct three processes Selector, Opter, and Chooser. In the mind, the processes are form bound contents or information entities (inner discrimination). Selector converts stimuli into percepts and selects the percepts for Opter. Opter chooses the courses of action of the processing the percepts and selects the processed percepts for Chooser. Chooser selects which of the percepts transform into overt responses. There also are variables, which define persons such as gender, socioeconomic status, and occupation. Therefore, it was necessary to include in the research gender, socioeconomic status, occupational class, and various collective bodies, where persons live. Consequentially, it was hypothesized that gender, socioeconomic status, occupational class, and social environment regulate action of Selector, Opter, and Chooser. Null hypothesis was no regulation occurs

\section{Variables and Their Operations}

Percepts (conscious, experiential processes resulting in perceptual processing) were the processes up whom other processes worked. The percepts comprised of socio-topics such as commerce, education, traffic, politics, industry, and healthcare.

Selector included in three variables 1) a participant answers the question at once, which means the percepts are finished 2) the participant pauses for a moment before answering, which means the percepts are in preparation 3) the participant begins to answer but interrupts, and continues, which means the percepts are unfinished.

Opter had six variables. The participant classifies or puts mind processes into categories. For example," there are such kind of persons as jerks, nerds, and players". When the participant chunks he or she puts the mind processes into heaps. For example," I should rather put persons into groups according to their capabilities". The participant orders, which 
means he or she arranges the mind processes properly or methodically. For example," if we put all the wooden pieces together, it will work". When the participant associates then he or she connects the mind processes. For example," John is her friend her friend is Moira so John is a friend of Moira". In modification, the participant changes over the mind processes. For example, "I prefer to say it in this way". The participant transmutes the mind processes, which means he or she changes the mind processes into completely different ones. For example, "you don't know the matter it goes through five phases".

Chooser included in six variables, too. Giving credence, expressing beliefs, viewing, informing, giving knowledge, and knowing-how. In giving credence, a participant believes in reports, statistics, and hearsay. In expressing beliefs, the participant assumes a state of affairs. In viewing, the participant has a personal comprehension. In informing the participant has facts to tell. In knowledge, the participant has organized information. In know-how, the participant has a command of action in reality. Attaching credence occurs when the participant refers to statistics in talking. For example, “According to Statistics Finland number of singles is growing”. Expressing beliefs is in question when the participant refers to a presumed state of affairs. For example, "It is probable life exists in Space". When the participant presents his or her personal comprehension it is the view. For example, "I see persons follow up with the laws of avidity, and of the least effort". The participant tells facts then informing occurs. For example," If you want to accomplish the task then following measures are to include". The participant arranges information in talking then it is knowledge. For example, "Photosynthesis produces oxygen in atmosphere". The participant is able to tell how things go, and how to manage with them, then it is knowinghow. For example, "First you wash the apple then peel it, remove the seed vessel, and cut in pieces like this". 
Social environment is in question when the participant refers to a person or to a community. The referents are an individual, a primary group (family), a secondary group (school), a political action group (a party), an organization (a firm), and population of people. The environmental referents are in the processes whereas the background variables are outside the processes. Gender is male, female. Socioeconomic groups followed up with the classification of Statistics Finland (1989): self-employed persons; upper level employees with

administrative, managerial, professional, and related occupations; lower level employees with administrative, and clerical occupations, and manual workers. Occupational classes comprised of leaders, experts, officers, and servants seen from the societal viewpoint. For example, the leaders in communes, the experts in research institutes, the officers in state bureaus, and the servants in post offices.

\section{Method}

\section{Participants}

The number of the participants was 186, 130 males, and 56 females. There were 34 self-employed persons; 47 upper level employees; 83 lower level employees, and 22 manual workers. The distribution of the occupational class was 68 leaders, 62 experts, 16 officers, and 40 servants.

\section{Procedure}

The participants participated in discussions, and the occasions were videotaped. Structured observation was the method of the data gathering with an observation minutes. The observation minutes included in the background information, and the variables in the rows. Time in steps ran along the rows. Each of the variables, and the social environments were tallied, separately in the time order of Selector, Opter, and Chooser. 
The social environments were tallied in spaces between the processes in the order of occurrence. The tallied observation minutes were added to one-one, which resulted in frequencies of the variables, and the social environments. The frequencies of the background variables formed in a usual way of adding the tallies.

\section{Results}

Table 1

Sum Frequencies of Process Variables

\begin{tabular}{llll}
\hline Variable & $\Sigma_{\mathrm{f}}$ & Variable & $0 \Sigma_{\mathrm{f}}$ \\
\hline Selector & & Chooser & \\
Percept finished & 1300 & Giving credence & 261 \\
Percept in preparation & 398 & Expressing beliefs & 58 \\
Percept unfinished & 89 & Viewing & 260 \\
Opter & & Informing & 230 \\
Classifies & 1533 & Giving knowledge & 51 \\
Chunks & 478 & Knowing-how & 559 \\
Orders & 976 & Social Environments & \\
Associates & 652 & Individual & 56 \\
Modifies & 919 & Primary group & 44 \\
Transmutes & 219 & Secondary group & 27 \\
& & Political activity group & 18 \\
& & Organization & 210 \\
& & Population & 115
\end{tabular}




\section{Reliability and Validity of Observation}

Duration of the variables in time steps varied. Therefore, it was necessary to partition frequency queues. The partitions included in the same number of rows in each mind process. The frequency queues were not even, and the rest of the queues was added to the last cell of the partition matrix. Otherwise, nulls replaced with the lacking frequencies. The means of the rows were obtained, and squared correlations were calculated between the means. The changes in the last cell were taken account in calculation of the means. Cronbach's $\alpha$ coefficient, and its square root were convenient to assess reliability.

Table 2

Cronbach's a-reliabilities of Processes Social Environments and Their Square Roots

\begin{tabular}{lll}
\hline Process & $\alpha$ & $\sqrt{ } \alpha$ \\
\hline Selector & 0.93 & 0.97 \\
Opter & 0.93 & 0.96 \\
Chooser & 0.91 & 0.95 \\
Social Environments & 0.97 & 0.98
\end{tabular}

In the place of validity, the frequency queues were partitioned according to the same number of the columns. The means, and their squared correlations were calculated with the same corrective measures as before with the rows. Thereafter, the squared correlations were subtracted from one, which resulted in coefficients of nondetermination. The means of the coefficients of nondetermination were obtained for the variables and the social environments.

Table 3

Means of Coefficients of Nondetermination of Processes and of Social Environments

\begin{tabular}{lc}
\hline Process & $\mathrm{k}^{2}$ \\
\hline Selector & 0.72 \\
Opter & 0.86 \\
Chooser & 0.80 \\
Social Environments & 0.82
\end{tabular}


The reliabilities are rather tolerable, and their square roots, too. The square roots of the $\alpha$-reliabilities are estimates of correlations with errorless observation. On the other hand, the variables, and the social environments are fairly separate because the coefficients of nondetermination indicate degree of absence of association between the variables. So, the observations are in the right categories, mostly.

\section{Elaboration and Analyses of Processes}

The maximum duration in time steps divided the sum scores of the process variables, Selector (93), Opter (96), and Chooser (89). Thereafter, the quotients were turned into probability vectors. Gender joined with the socioeconomic status, and joined with the occupational class for the sake of compatibility of the matrices. The sum scores of the social environments as such were converted into a probability vector.

Table 4

Probabilities of Background Variables

\begin{tabular}{lll}
\hline Background & Variable & $\mathrm{P}$ \\
\hline \multirow{2}{*}{ Gender } & Male & .35 \\
\multirow{2}{*}{ Socioeconomic status } & Female & .15 \\
& Self-employee & .09 \\
& Upper level employees & .13 \\
& Lower level employees & .22 \\
Gender & Manual workers & .06 \\
\multirow{3}{*}{ Occupational class } & Male & .35 \\
& Female & .15 \\
& Leaders & .18 \\
& Experts & .17 \\
& Officers & .11
\end{tabular}

Process Variable and Probability

Selector: Percepts finished .73 Percepts in preparation .22 Percepts unfinished .05 Opter: Classifies .32 Chunks .10 Orders .20 Associates .14 Modifies .19 Transmutes .05 Chooser: Credence .18 Belief .04 View .18 Information .16 Knowledge .04 Know-how .39 Environments: Individual .12 primary group .09 secondary group .06 political activity group .04 organization .45 population .24 
Cartesian products were formed from the probability vectors without the background variables. The vector of Selector was dimension 1 by 3. Therefore, the Selector vector was doubled because of the matrix of Selector to be compatible with other matrices. Sampling Without Replacement was applied to the Cartesian matrices because of the time order of the processes, Selector, Opter, and Chooser. The Selector matrix included in two 3 by 3 diagonal blocks with nulls in off-diagonal cells. The total sum of the result matrices was scaled down one. Bayes's formula was applicable to the columns of the result matrices. After that, squared orthonormal bases were derived from the Bayesian matrices, which resulted in double-stochastic matrices. Elaboration took place in groups of three variables in the double stochastic matrices with the formula 1.

$\mathrm{P}_{\mathrm{p}}=\operatorname{Sqrt}\left[\left(\mathrm{p} 1-\left(\mathrm{p} 2 * \mathrm{p} 3+\mathrm{p} 1{ }^{*} \mathrm{p} 2 * \mathrm{p} 3\right)\right)^{2} /(((\mathrm{p} 1+\mathrm{p} 2+\mathrm{p} 3)-(\mathrm{p} 1 * \mathrm{p} 2-\mathrm{p} 1 * \mathrm{p} 3-\mathrm{p} 2 * \mathrm{p} 3+\mathrm{p} 1 * \mathrm{p} 2 * \mathrm{p} 3))-\right.$ $\left.(\mathrm{p} 2 * \mathrm{p} 3+\mathrm{p} 1 * \mathrm{p} 2 * \mathrm{p} 3))^{2}\right]$; If $\left[0<=\mathrm{P}_{\mathrm{p}}<=1, \mathrm{P}_{\mathrm{p}}\right.$, else ="out of range" $]$

The left side of the equation is the partial probability between the variables one and two, and two and one when variable three was eliminated. There are two probabilities because of asymmetry. The markings $\mathrm{p} 1, \mathrm{p} 2$, and $\mathrm{p} 3$ meant conditional probabilities between variables. The formula is modifiable to scrutinize other relationships by changing the places between the probabilities. Elaboration was not over after elimination of the variables. Therefore, it was necessary to calculate the eliminated probabilities anew to correspond with the partial probabilities. The formula of percentage change was suitable for the purpose without multiplication by one-hundred. Elimination of one variable may lower or rise the probabilities of other variables. When a probability lowers or vanishes because of elimination, a new probability is calculative after elaboration with formula 2 
$\mathrm{Xl}=\mathrm{a}+(((1-\mathrm{a})) *((\mathrm{~b}-\mathrm{c}) / \mathrm{b}))$

The left side of the formula ( $\mathrm{xl})$ is the new probability, and a is the eliminated probability.

The latter part of the formula is the change coefficient, and the multiplier is the complement of the old probability, a controller that takes care of not exceeding one. When a probability rises because of elimination then the event is inverse to the preceding one. So, the symbols change their places like in the formula 3.

$\mathrm{Xr}=\mathrm{a}+(((1-\mathrm{a})) *((\mathrm{c}-\mathrm{b}) / \mathrm{c}))$

The Xr-marking is the new probability. The former case presents extraneous variables, for example, the latter one hold-back variables, which keep probabilities low or null.

After elaboration, the new probabilities formed causal matrices, from which the orthonormal matrices derived with values over 0.1 , and the row sums as ones. Orthogonality test had its place in this context. The matrices included in the start causalities for dynamic.

Table 5

Results of Orthogonality Test

\begin{tabular}{lllll}
\hline Processes & MP & GSE & GO & ENV \\
\hline Selector & .65 & .65 & .61 & .65 \\
Opter & .62 & .74 & .72 & .74 \\
Chooser & .59 & .78 & .60 & .50
\end{tabular}

Note. MP means mere process without the background variables; GSE means gender, and socioeconomic status; GO means gender, and occupational class, and ENV is the environmental variables. The values are below one. 
The background, and the environmental probability vectors multiplied the column probability vectors of Selector, Opter, and Chooser. The product vectors formed Cartesian products with the mere row vectors of Selector, Opter, and Chooser. Thereafter, the same procedure as with the mere process applied to other processes. The percept (topics) vector was scaled to one. The stimulus vector started at $t^{0}$. and the causal matrices started from $\mathrm{t}^{1}$. The stimulus vector left multiplied the first causal matrix, which produced the percept output that acted as input for the next powered matrix. After that output acted as input to next process. Every time the powers of the stimulus-percept vector, and the causal matrix increased by one. In this way, a sequence of loops emerged: The last percept vector of selector acted as input to Opter, and the last output of Opter acted as input to Chooser. The loops in Selector, Opter, and Chooser ended when the percept vector returned to its start state or it repeated itself.

\section{Probabilistic Causal Processes}

Table 6 includes in the start matrices of the processes. The abbreviations are as follows. Background: $\mathrm{M}=$ men, $\mathrm{W}=$ women, $\mathrm{See}=$ self-employed persons, $\mathrm{Upl}=$ upper level employees, $\mathrm{Lol}=$ lower level employees, $\mathrm{Mw}=$ manual workers; Lea = leaders, $\mathrm{Exp}=$ experts, Off = officers, Ser = servants; Environments: $\mathrm{I}=$ individual, $\mathrm{Pg}=$ primary group, $\mathrm{Sg}$ $=$ secondary group, $\mathrm{Pag}=$ political activity group, $\mathrm{Org}=$ organization, and $\mathrm{Pop}=$ population. Percepts: $\mathrm{Com}=$ commerce, $\mathrm{Edu}=$ education, Tra $=$ traffic, Pol $=$ politics, Ind $=$ industry, Hea $=$ healthcare; Selector. $\mathrm{pf}=$ percepts finished, $\mathrm{pip}=$ percepts in preparation, $\mathrm{puf}=$ percepts unfinished; Opter: cla $=$ classifies, $c h u=$ chunks, ord $=$ orders, ass $=$ associates, $\bmod$ $=$ modifies, $\operatorname{tra}=$ transmutes; Chooser: cre $=$ credence, bel $=$ belief, vie $=$ view, inf $=$ information, $\mathrm{kno}=$ knowledge, $\mathrm{knh}=$ knowhow; $\mathrm{MP}=$ mere process, $\mathrm{GSE}=$ gender and socioeconomic group process, $\mathrm{GO}=$ gender and occupational class process, and $\mathrm{ENV}=$ environmental process. 
Table 6

Start Matrices of Processes' Selector Opter and Chooser

\begin{tabular}{|c|c|c|c|c|c|c|c|c|c|c|c|c|c|c|c|c|c|}
\hline MP & & Selector & & & & & & & & & Opter & & & & & & \\
\hline$\underline{\mathrm{T}^{0}}$ & $\underline{\mathrm{P}}$ & $\underline{T}^{1}$ & pf & pip & puf & pf & pip & puf & $\underline{\mathrm{T}^{0}}$ & $\underline{\mathrm{P}}$ & ${\underline{\mathrm{T}^{1}}}^{-1}$ & cla & $\underline{\text { chu }}$ & ord & ass & $\underline{\bmod }$ & tra \\
\hline$\overline{\mathrm{Com}}$ & .12 & $\overline{\mathrm{pf}}$ & .12 & .41 & .47 & 0 & 0 & 0 & $\overline{\mathrm{Com}}$ & & $\overline{\mathrm{cla}}$ & $\overline{0}$ & $\overline{0}$ & $\overline{1 .}$ & $\overline{0}$ & $\overline{0}$ & $\overline{0}$ \\
\hline$\overline{\mathrm{Edu}}$ & .23 & pip & .39 & .15 & .46 & 0 & 0 & 0 & $\underline{E d u}$ & & $\underline{\operatorname{chu}}$ & 0 & 0 & 0 & 0 & .50 & .50 \\
\hline$\underline{\text { Tra }}$ & .05 & puf & .53 & .47 & 0 & 0 & 0 & 0 & Tra & & ord & 0 & 0 & 0 & 1. & 0 & 0 \\
\hline Pol & .30 & pf & 0 & 0 & 0 & .15 & .27 & .58 & $\underline{\mathrm{Pol}}$ & & $\underline{\text { ass }}$ & .52 & .48 & 0 & 0 & 0 & 0 \\
\hline Ind & .05 & pip & 0 & 0 & 0 & .59 & .41 & 0 & Ind & & mod & 0 & 0 & 0 & 0 & .49 & .51 \\
\hline Hea & .25 & puf & 0 & 0 & 0 & .26 & .32 & .42 & Hea & & tra & .36 & .47 & .17 & 0 & 0 & 0 \\
\hline & & Chooser & & & & & & & GSE & & Selector & & & & & & \\
\hline$\underline{\mathrm{T}^{0}}$ & $\underline{P}$ & $\underline{\mathrm{T}^{1}}$ & cre & bel & vie & inf & kno & $\mathrm{knh}$ & $\underline{\mathrm{T}^{0}}$ & $\underline{P}$ & $\underline{\mathrm{T}^{1}}$ & $\mathrm{pf}$ & pip & puf & pf & pip & puf \\
\hline Com & & cre & $\overline{0}$ & $\overline{0}$ & .23 & .66 & $\overline{0}$ & .11 & Com & .12 & $\underline{\bar{M}}$ & .12 & .40 & .47 & 0 & 0 & 0 \\
\hline$\underline{\mathrm{Edu}}$ & & bel & 0 & 0 & 0 & 0 & .76 & .24 & $\overline{\text { Edu }}$ & .23 & $\underline{\mathrm{W}}$ & .40 & .15 & .45 & 0 & 0 & 0 \\
\hline$\underline{\text { Tra }}$ & & $\underline{\mathrm{vie}}$ & 0 & 0 & 0 & .19 & .21 & .60 & $\overline{\text { Tra }}$ & .05 & $\overline{\text { See }}$ & .51 & .49 & 0 & 0 & 0 & 0 \\
\hline$\overline{\mathrm{Pol}}$ & & $\overline{\text { inf }}$ & .64 & 0 & .23 & .13 & 0 & 0 & $\overline{\mathrm{Pol}}$ & .30 & $\overline{\mathrm{Upl}}$ & 0 & 0 & 0 & 0 & .63 & .37 \\
\hline$\underline{\text { Ind }}$ & & kno & 0 & 1. & 0 & 0 & 0 & 0 & $\underline{\underline{I n d}}$ & .05 & $\overline{\mathrm{Lol}}$ & 0 & 0 & 0 & .69 & 0 & .31 \\
\hline Hea & & $\overline{\mathrm{knh}}$ & .35 & 0 & .52 & 0 & 0 & .13 & $\overline{\text { Hea }}$ & .25 & $\overline{\mathrm{MW}}$ & 0 & 0 & 0 & .22 & .42 & .36 \\
\hline & & $\overline{\text { Opter }}$ & & & & & & & & & Chooser & & & & & & \\
\hline$\underline{\mathrm{T}^{0}}$ & $\underline{P}$ & $\frac{\underline{T}^{1}}{}$ & cla & $\underline{\text { chu }}$ & ord & $\underline{\text { ass }}$ & $\underline{\bmod }$ & $\underline{\text { tra }}$ & $\underline{\mathrm{T}^{0}}$ & $\underline{P}$ & ${\underline{\mathrm{T}^{1}}}$ & cre & bel & vie & $\underline{\text { inf }}$ & kno & $\mathrm{knh}$ \\
\hline Com & & $\underline{\bar{M}}$ & .36 & $\overline{0}$ & $\overline{0}$ & $\overline{0}$ & .64 & $\overline{0}$ & Com & & $\underline{\bar{M}}$ & .53 &.$\overline{47}$ & $\overline{0}$ & $\overline{0}$ & $\overline{0}$ & 0 \\
\hline$\underline{E \mathrm{Edu}}$ & & $\underline{\mathrm{W}}$ & .19 & 0 & 0 & 0 & 0 & .81 & $\overline{\text { Edu }}$ & & $\underline{\mathrm{W}}$ & 0 & 0 & 0 & 0 & .50 & .50 \\
\hline$\overline{\mathrm{Tra}}$ & & See & 0 & .23 & 0 & .77 & 0 & 0 & $\underline{\text { Tra }}$ & & See & 0 & 0 & 0 & .56 & .16 & .28 \\
\hline $\mathrm{Pol}$ & & $\underline{\mathrm{Upl}}$ & .12 & .61 & .27 & 0 & 0 & 0 & $\overline{\mathrm{Pol}}$ & & $\underline{\mathrm{Upl}}$ & 0 & 0 & .87 & .13 & 0 & 0 \\
\hline$\overline{\text { Ind }}$ & & $\overline{\mathrm{Lol}}$ & 0 & .24 & .47 & .28 & 0 & 0 & Ind & & Lol & .17 & .20 & 0 & .29 & .23 & .11 \\
\hline Hea & & $\underline{\mathrm{Mw}}$ & .32 & 0 & .22 & 0 & .18 & .28 & $\underline{\overline{\mathrm{Hea}}}$ & & $\overline{\mathrm{Mw}}$ & .29 & .33 & .11 & 0 & .12 & .14 \\
\hline $\mathrm{GO}$ & & Selector & & & & & & & & & Opter & & & & & & \\
\hline
\end{tabular}

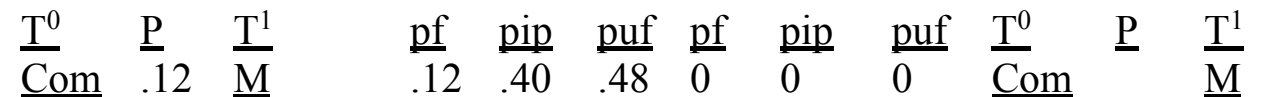

$\begin{array}{lllllllllll}\underline{\underline{\mathrm{Edu}}} & .23 & \underline{\mathrm{W}} & .41 & .14 & .45 & 0 & 0 & 0 & \underline{\mathrm{Edu}} & \underline{\mathrm{W}}\end{array}$

$\underline{\text { cla }} \underline{\text { chu }} \underline{\text { ord }} \underline{\text { ass }} \underline{\text { mod }} \underline{\text { tra }}$

$\underline{\underline{T r a}} \quad .05 \quad \underline{\text { Lea }}$

$\begin{array}{lllllll}.50 & .50 & 0 & 0 & 0 & 0 & \frac{\mathrm{Tra}}{\mathrm{Tra}}\end{array}$

$\underline{\text { Lea }}$

1. $00 \begin{array}{llll}0 & 0 & 0 & 0\end{array}$

$\underline{\underline{P o l}} \quad .30 \quad \underline{\text { Exp }}$

$\begin{array}{lllllll}0 & 0 & 0 & .15 & .28 & .56 & \underline{\underline{\mathrm{Pol}}}\end{array}$

$\underline{\operatorname{Exp}}$

$\begin{array}{llllll}0 & 0 & 0 & 0 & .50 & .50\end{array}$

$\underline{\underline{\text { Ind }}} \quad .05 \quad \underline{\underline{\text { Off }}}$

$\begin{array}{lllllll}0 & 0 & 0 & .70 & .30 & 0 & \underline{\text { Ind }}\end{array}$

$\underline{\underline{\text { Off }}}$

$\begin{array}{llllll}0 & .13 & 0 & .87 & 0 & 0\end{array}$

$\underline{\text { Hea }} .25 \underline{\text { Ser }}$

$\begin{array}{lllllll}0 & 0 & 0 & .14 & .42 & .44 & \underline{\text { Hea }}\end{array}$

$\underline{\underline{\text { Ser }}}$

$\begin{array}{llllll}0 & .54 & .26 & .20 & 0 & 0\end{array}$

Chooser

ENV

$\underline{\text { Selector }}$

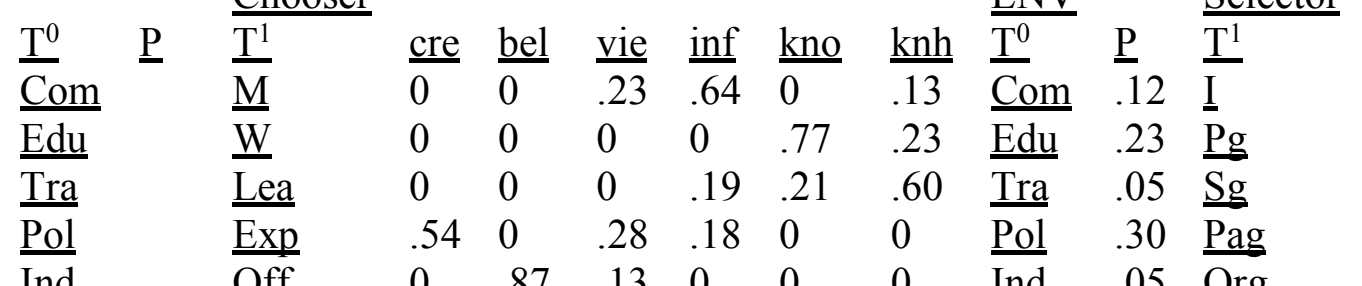

$\underline{\underline{\text { Ind }}} \quad \begin{array}{llllllllll}\underline{\text { Off }} & 0 & .87 & .13 & 0 & 0 & 0 & \underline{\underline{\text { Ind }}} & .05 & \underline{\mathrm{Org}}\end{array}$

\begin{tabular}{lllllllllll} 
Hea & $\underline{\text { Ser }}$ & .57 & 0 & .43 & 0 & 0 & 0 & $\underline{\text { Hea }}$ & .25 & $\underline{\underline{\text { Pop }}}$ \\
\hline Chooser
\end{tabular}

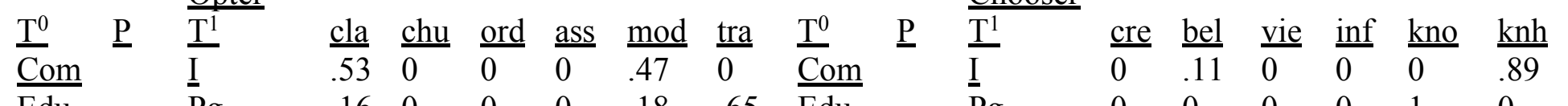

$\begin{array}{lllllllll}\mathrm{Edu} & \mathrm{Pg} & .16 & 0 & 0 & 0 & .18 & .65 & \underline{\mathrm{Ed} u}\end{array}$

$\begin{array}{lllllllll}\text { Tra } & \underline{\mathrm{Sg}} & 0 & .12 & 0 & .88 & 0 & 0 & \underline{\mathrm{Tra}}\end{array}$

$\begin{array}{lllllllll}\underline{\mathrm{Pol}} & \underline{\mathrm{Pag}} & .11 & .33 & .44 & 0 & .12 & 0 & \underline{\mathrm{Pol}}\end{array}$

$\begin{array}{lllllllll}\underline{\underline{\text { Ind }}} & \underline{\mathrm{Org}} & 0 & .43 & .40 & .17 & 0 & 0 & \underline{\mathrm{Ind}} \\ \mathrm{Hea} & \mathrm{Pop} & .22 & .19 & 0 & 0 & .25 & .34 & \mathrm{Hea}\end{array}$

$\underline{\mathrm{Pg}}$

$\begin{array}{llllll}0 & .20 & .38 & 0 & .28 & .14\end{array}$

$\begin{array}{llllll}0 & .14 & .26 & 0 & .23 & .37\end{array}$

$\underline{\text { Hea }}$

pf pip puf pf pip puf

$\begin{array}{llllll}.12 & .40 & .47 & 0 & 0 & 0\end{array}$

$\begin{array}{llllll}.29 & .54 & .16 & 0 & 0 & 0\end{array}$

$\begin{array}{llllll}.62 & 0 & .38 & 0 & 0 & 0\end{array}$

$\begin{array}{llllll}0 & 0 & 0 & .13 & .25 & .62\end{array}$

$\begin{array}{llllll}0 & 0 & 0 & .46 & .54 & 0\end{array}$

$\begin{array}{llllll}0 & 0 & 0 & .42 & .22 & .36\end{array}$

$\begin{array}{lllllll}\overrightarrow{\mathrm{Sg}} & .15 & 0 & 0 & .85 & 0 & 0\end{array}$

$\begin{array}{lllllll}\mathrm{Pag} & .80 & 0 & 0 & .20 & 0 & 0\end{array}$

$\begin{array}{lllllll}\overline{\mathrm{Org}} & 0 & .52 & .48 & 0 & 0 & 0\end{array}$ 
Although, causation after elaboration was solved, it was necessary to focus on the specifics of the background processes. The start matrices of the mere process applied to the criteria as in Table 6. Only those probabilities, which exceeded the probabilities of the mere processes included in the specificity analysis. The probabilities being less than or equal to the probabilities of the mere processes lead to infer that no causation or no causation and causation, at the same time. Therefore, the selection of the greater probabilities was reasonable. In Table7, there are the background processes.

Table 7

Background Processes

Gender and Socioeconomic Group Process

\section{$\underline{\text { Selector }}$}

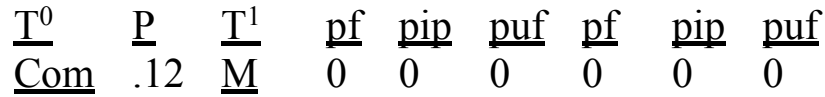
$\begin{array}{lllllllll}\underline{\mathrm{Edu}} & .23 & \underline{\mathrm{W}} & 1 . & 0 & 0 & 0 & 0 & 0\end{array}$

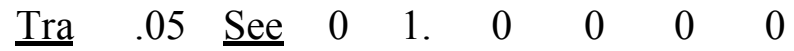

$\begin{array}{lllllllll}\text { Pol } & .30 & \underline{\mathrm{Upl}} & 0 & 0 & 0 & 0 & 1 . & 0\end{array}$

$\begin{array}{lllllllll}\underline{\text { Ind }} & .05 & \underline{\mathrm{Lol}} & 0 & 0 & 0 & .69 & 0 & .31\end{array}$

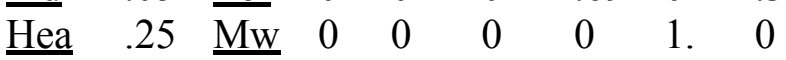

$\begin{array}{lllllllll}\frac{\mathrm{T}^{1}}{\mathrm{Com}} & \underline{\mathrm{P}} & \frac{\mathrm{T}^{2}}{23} & \underline{\mathrm{pf}} & \underline{\mathrm{pip}} & \underline{\text { puf }} & \underline{\mathrm{pf}} & \underline{\text { pip }} & \underline{\text { puf }}\end{array}$ $\begin{array}{lllllllll}\underline{\mathrm{Edu}} & .05 & \underline{\mathrm{W}} & 0 & 0 & 0 & 0 & 0 & 0\end{array}$ $\begin{array}{lllllllll}\text { Tra } & 0 & \underline{\text { See }} & 1 . & 0 & 0 & 0 & 0 & 0\end{array}$ $\begin{array}{lllllllll}\underline{\text { Pol }} & .03 & \underline{\text { Upl }} & 0 & 0 & 0 & .69 & 0 & .31\end{array}$

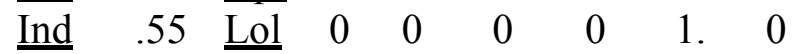
$\begin{array}{lllllllll}\underline{\underline{\mathrm{Hea}}} & .02 & \underline{\mathrm{Mw}} & 0 & 0 & 0 & .69 & 0 & .31\end{array}$

$\begin{array}{lllllllll}\frac{\mathrm{T}^{2}}{\mathrm{Com}} & \underline{\mathrm{P}} & \underline{\mathrm{T}^{3}} & \underline{\mathrm{pf}} & \underline{\mathrm{pip}} & \underline{\mathrm{puf}} & \underline{\mathrm{pf}} & \underline{\mathrm{pip}} & \underline{\mathrm{puf}}\end{array}$ $\begin{array}{lllllllll}\underline{\mathrm{Edu}} & 0 & \underline{\mathrm{W}} & 0 & 0 & 0 & 0 & 0 & 0\end{array}$ $\begin{array}{lllllllll}\underline{\text { Tra }} & 0 & \underline{\text { See }} & 0 & 0 & 0 & 0 & 0 & 0\end{array}$

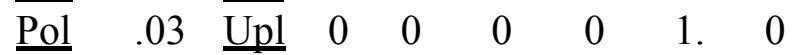
$\begin{array}{lllllllll}\underline{\text { Ind }} & .55 & \underline{\mathrm{Lol}} & 0 & 0 & 0 & .69 & 0 & .31\end{array}$ $\begin{array}{lllllllll}\text { Hea } & 02 & \underline{\mathrm{Mw}} & 0 & 0 & 0 & 0 & 1 . & 0\end{array}$
$\begin{array}{lllllllll}\frac{\mathrm{T}^{3}}{\mathrm{Com}} & \underline{\mathrm{P}} & \underline{\mathrm{T}^{4}} & \underline{\mathrm{pf}} & \underline{\mathrm{pip}} & \underline{\mathrm{puf}} & \underline{\mathrm{pf}} & \underline{\mathrm{pip}} & \underline{\mathrm{puf}}\end{array}$

$\begin{array}{lllllllll}\underline{\mathrm{Edu}} & 0 & \underline{\mathrm{W}} & 0 & 0 & 0 & 0 & 0 & 0\end{array}$

$\begin{array}{lllllllll}\underline{\text { Tra }} & 0 & \underline{\text { See }} & 0 & 0 & 0 & 0 & 0 & 0\end{array}$

$\begin{array}{lllllllll}\underline{\underline{\mathrm{Pol}}} & .38 & \underline{\mathrm{Upl}} & 0 & 0 & 0 & .69 & 0 & .31\end{array}$

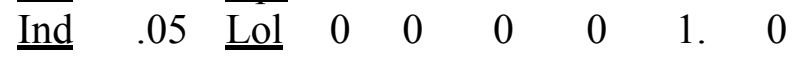

$\begin{array}{lllllllll}\text { Hea } & .17 & \underline{\mathrm{Mw}} & 0 & 0 & 0 & .69 & 0 & .31\end{array}$

\section{$\underline{\text { Opter }}$}

$\begin{array}{lllllllll}\frac{\mathrm{T}^{0}}{\mathrm{Com}} & \underline{\mathrm{P}} & \underline{\mathrm{T}^{1}} & \underline{\mathrm{cla}} & \underline{\mathrm{chu}} & \underline{\mathrm{ord}} & \underline{\mathrm{ass}} & \underline{\mathrm{mod}} & \underline{\mathrm{tra}}\end{array}$ $\begin{array}{lllllllll}\underline{\underline{\mathrm{Edu}}} & 0 & \underline{\mathrm{W}} & .19 & 0 & 0 & 0 & 0 & .81\end{array}$

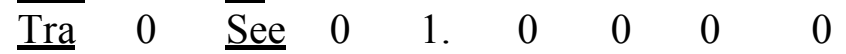
$\begin{array}{lllllllll}\underline{\underline{\mathrm{Pol}}} & .38 & \underline{\mathrm{Upl}} & 0 & .69 & .31 & 0 & 0 & 0\end{array}$ $\begin{array}{lllllllll}\underline{\text { Ind }} & .05 & \underline{\text { Lol }} & 0 & .24 & .47 & .28 & 0 & 0\end{array}$ $\begin{array}{lllllllll}\text { Hea } & .17 & \underline{\mathrm{Mw}} & 0 & 0 & .32 & 0 & .26 & .41\end{array}$

$\begin{array}{lllllllll}\mathrm{T}^{1} & \frac{\mathrm{P}}{\mathrm{Com}} & \frac{\mathrm{T}^{2}}{\mathrm{M}} & \frac{\mathrm{cla}}{.13} & \frac{\text { chu }}{.16} & \frac{\text { ord }}{.30} & \frac{\text { ass }}{.18} & \frac{\bmod }{.23} & \frac{\text { tra }}{0}\end{array}$

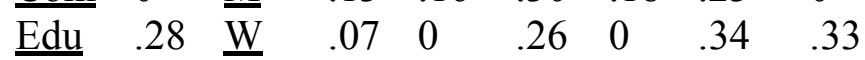
$\begin{array}{lllllllll}\text { Tra } & .20 & \underline{\text { See }} & .19 & 0 & 0 & 0 & 0 & .81\end{array}$ $\begin{array}{lllllllll}\underline{\mathrm{Pol}} & .01 & \underline{\mathrm{Upl}} & .13 & .31 & 0 & 0 & 0 & .56\end{array}$ $\begin{array}{lllllllll}\underline{\text { Ind }} & .05 & \underline{\text { Lol }} & .05 & .67 & .09 & 0 & 0 & .20\end{array}$

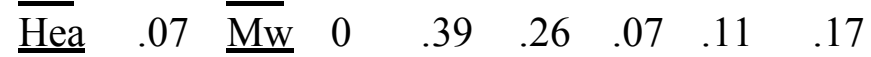




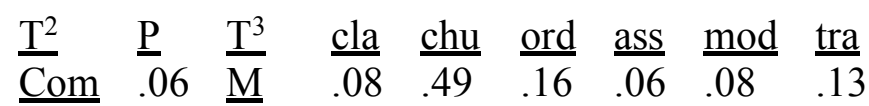
$\begin{array}{lllllllll}\underline{\mathrm{Edu}} & .06 & \underline{\mathrm{W}} & .02 & .34 & .27 & .10 & .13 & .14\end{array}$ $\begin{array}{lllllllll}\underline{\text { Tra }} & .09 & \underline{\text { See }} & .07 & 0 & .26 & 0 & .34 & .33\end{array}$ $\begin{array}{lllllllll}\underline{\mathrm{Pol}} & .01 & \underline{\mathrm{Upl}} & .11 & 0 & .18 & 0 & .23 & .48\end{array}$ $\begin{array}{lllllllll}\underline{\text { Ind }} & .10 & \underline{\underline{L o l}} & .14 & .09 & .06 & 0 & .08 & .62\end{array}$ $\begin{array}{lllllllll}\underline{\mathrm{Hea}} & .28 & \underline{\mathrm{Mw}} & .07 & .34 & .13 & .03 & .04 & .38\end{array}$

$\underline{\mathrm{T}^{3}} \quad \underline{\mathrm{P}} \quad \underline{\mathrm{T}^{4}} \quad \underline{\text { cla }} \quad \underline{\text { chu }}$ ord $\underline{\text { ass }} \underline{\bmod } \underline{\text { tra }}$ $\begin{array}{lllllllll}\overline{\mathrm{Com}} & .05 & \underline{\mathrm{M}} & .12 & .23 & .10 & .02 & .08 & .44\end{array}$ $\begin{array}{lllllllll}\mathrm{Edu} & .15 & \underline{\mathrm{W}} & .07 & .37 & .14 & .04 & .05 & .33\end{array}$ $\begin{array}{lllllllll}\underline{\text { Tra }} & .09 & \underline{\text { See }} & .02 & .34 & .27 & .10 & .13 & .14\end{array}$ $\begin{array}{lllllllll}\underline{\underline{P} \mathbf{p l}} & .02 & \underline{\mathrm{Upl}} & .04 & .24 & .27 & .07 & .19 & .20\end{array}$ $\begin{array}{lllllllll}\text { Ind } & .07 & \underline{\text { Lol }} & .07 & .08 & .24 & .02 & .26 & .33\end{array}$

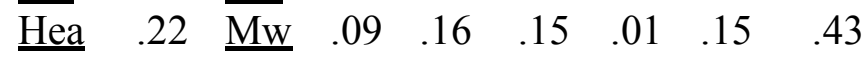

$\underline{\mathrm{T}^{4}} \quad \underline{\mathrm{P}} \quad \underline{\mathrm{T}}^{5} \quad \underline{\text { cla }} \quad \underline{\text { chu }} \quad$ ord $\quad \underline{\text { ass }} \quad \underline{\bmod }$ tra

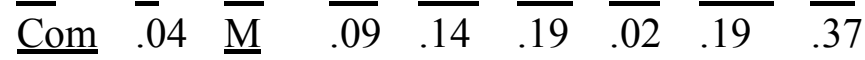
$\begin{array}{lllllllll}\mathrm{Edu} & .14 & \underline{\mathrm{W}} & .10 & .17 & .14 & .01 & .14 & .43\end{array}$ $\begin{array}{lllllllll}\underline{\text { Tra }} & .11 & \underline{\text { See }} & .07 & .37 & .14 & .04 & .05 & .33\end{array}$ $\begin{array}{lllllllll}\underline{\text { Pol }} & .02 & \underline{\mathrm{Upl}} & .06 & .36 & .18 & .05 & .08 & .27\end{array}$ $\begin{array}{lllllllll}\underline{\text { Ind }} & .08 & \underline{\text { Lol }} & .04 & .32 & .24 & .07 & .13 & .20\end{array}$ $\begin{array}{lllllllll}\underline{\text { Hea }} & .21 & \underline{\mathrm{Mw}} & .06 & .20 & .21 & .04 & .17 & .31\end{array}$

\section{Chooser}

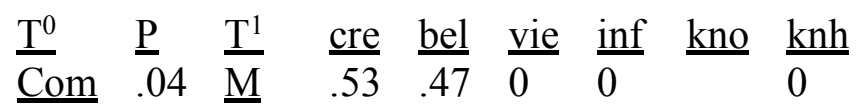
$\begin{array}{lllllllll}\underline{\mathrm{Edu}} & .14 & \underline{\mathrm{W}} & 0 & 0 & 0 & 0 & 0 & 1 .\end{array}$ $\begin{array}{lllllllll}\underline{\text { Tra }} & .11 & \underline{\text { See }} & 0 & 0 & 0 & 1 . & 0 & 0\end{array}$ $\begin{array}{lllllllll}\underline{\mathrm{Pol}} & .02 & \underline{\mathrm{Upl}} & 0 & 0 & 1 . & 0 & 0 & 0\end{array}$ $\begin{array}{lllllllll}\underline{\text { Ind }} & .08 & \underline{\text { Lol }} & .21 & 0 & 0 & .36 & .29 & .14\end{array}$ $\begin{array}{lllllllll}\underline{\underline{\text { Hea }}} & .20 & \underline{\mathrm{Mw}} & 0 & .56 & 0 & 0 & .20 & .24\end{array}$

$\underline{\mathrm{T}^{1}} \quad \underline{\mathrm{P}} \quad \underline{\mathrm{T}^{2}} \quad \underline{\text { cre }} \quad \underline{\text { bel }} \quad \underline{\text { vie }} \underline{\underline{\text { inf }}} \underline{\mathrm{kno}} \underline{\mathrm{knh}}$

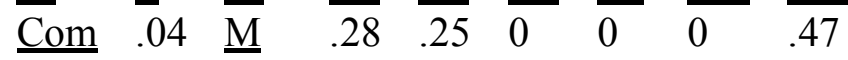
$\begin{array}{lllllllll}\underline{\underline{\mathrm{Edu}}} & .13 & \underline{\mathrm{W}} & 0 & .56 & 0 & 0 & .20 & .24\end{array}$ $\begin{array}{lllllllll}\underline{\underline{T r a}} & .02 & \underline{\text { See }} & 0 & 0 & 1 . & 0 & 0 & 0\end{array}$ $\begin{array}{lllllllll}\underline{\underline{P o l}} & .14 & \underline{\mathrm{Upl}} & 0 & 0 & 0 & 1 . & 0 & 0\end{array}$ $\begin{array}{lllllllll}\underline{\underline{\text { Ind }}} & .06 & \underline{\mathrm{Lol}} & .17 & .18 & .36 & .10 & .11 & .07\end{array}$ $\begin{array}{lllllllll}\underline{\underline{\text { Hea }}} & .20 & \underline{\mathrm{Mw}} & .04 & .13 & 0 & .07 & .11 & .64\end{array}$
$\begin{array}{lllllllll}\mathrm{T}^{2} & \underline{\mathrm{P}} & \underline{\mathrm{T}^{3}} & \underline{\mathrm{cre}} & \text { bel } & \text { vie } & \underline{\mathrm{inf}} & \frac{\mathrm{kno}}{\mathrm{C}} & \frac{\mathrm{knh}}{.15}\end{array}$ $\begin{array}{lllllllll}\underline{\underline{E d u}} & .12 & \underline{\mathrm{W}} & .04 & .13 & 0 & .07 & .11 & .64\end{array}$ $\begin{array}{lllllllll}\underline{\mathrm{Tra}} & .05 & \underline{\mathrm{See}} & 0 & 0 & 0 & 1 . & 0 & 0\end{array}$ $\begin{array}{lllllllll}\underline{\mathrm{Pol}} & .16 & \underline{\mathrm{Upl}} & 0 & 0 & 1 . & 0 & 0 & 0\end{array}$ $\begin{array}{lllllllll}\underline{\underline{\text { Ind }}} & .06 & \underline{\mathrm{Lol}} & .12 & .12 & .10 & .40 & .05 & .21\end{array}$ $\begin{array}{lllllllll}\underline{\text { Hea }} & .18 & \underline{\mathrm{Mw}} & .05 & .38 & .07 & .04 & .16 & .30\end{array}$

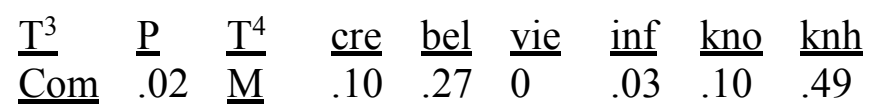
$\begin{array}{lllllllll}\overline{\mathrm{Edu}} & .11 & \mathrm{~W} & .05 & .38 & .07 & .04 & .16 & .30\end{array}$

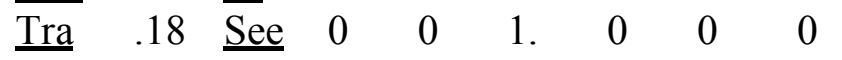
$\begin{array}{lllllllll}\underline{\underline{P o l}} & .08 & \underline{\mathrm{Upl}} & 0 & 0 & 0 & 1 . & 0 & 0\end{array}$

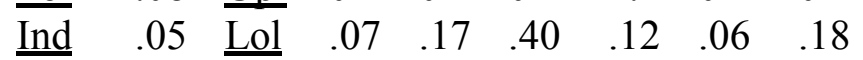

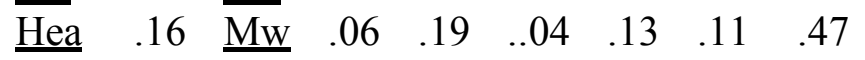

$\begin{array}{lllllllll}\mathrm{T}^{4} & \underline{\mathrm{P}} & \mathrm{T}^{1} & \underline{\text { cre }} & \text { bel } & \text { vie } & \underline{\text { inf }} & \underline{\mathrm{kno}} & \mathrm{knh} \\ \underline{\mathrm{Com}} & .02 & \underline{\mathrm{M}} & \frac{.07}{.32} & \frac{.03}{.04} & \frac{.13}{.40}\end{array}$ $\begin{array}{lllllllll}\underline{\mathrm{Edu}} & .08 & \underline{\mathrm{W}} & .06 & .19 & .04 & .13 & .11 & .47\end{array}$

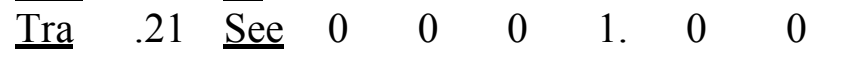
$\underline{\underline{\mathrm{Pol}}} \quad \begin{array}{llllllll}.12 & \underline{\mathrm{Upl}} & 0 & 0 & 1 . & 0 & 0 & 0\end{array}$ $\begin{array}{lllllllll}\underline{\underline{\text { Ind }}} & .04 & \underline{\mathrm{Lol}} & .05 & .13 & .12 & .42 & .05 & .22\end{array}$ $\begin{array}{lllllllll}\text { Hea } & .13 & \underline{\mathrm{Mw}} & .05 & .29 & .13 & .08 & .13 & .32\end{array}$

$\frac{\mathrm{T}^{5}}{\mathrm{C}} \quad \frac{\mathrm{P}}{02} \quad \frac{\mathrm{T}^{6}}{\mathrm{M}} \quad \frac{\mathrm{cre}}{06} \quad \frac{\text { bel }}{26} \quad \frac{\text { vie }}{04} \quad \frac{\text { inf }}{08} \quad \frac{\mathrm{kno}}{12} \frac{\mathrm{knh}}{44}$ $\begin{array}{lllllllll}\overline{\mathrm{Edu}} & .06 & \underline{\mathrm{W}} & .05 & .29 & .13 & .08 & .13 & .32\end{array}$

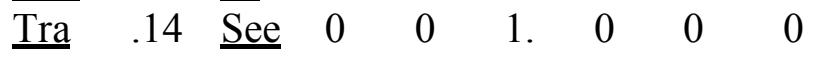
$\begin{array}{lllllllll}\underline{\underline{P o l}} & .25 & \underline{\mathrm{Upl}} & 0 & 0 & 0 & 1 . & 0 & 0\end{array}$ $\begin{array}{lllllllll}\underline{\text { Ind }} & .03 & \underline{\text { Lol }} & .04 & .15 & .42 & .14 & .06 & .19\end{array}$ $\begin{array}{lllllllll}\underline{\text { Hea }} & .10 & \underline{\mathrm{Mw}} & .06 & .20 & .08 & .18 & .10 & .39\end{array}$

$\underline{\text { Com Edu }} \underline{\text { Tra }} \underline{\text { Pol Ind }} \underline{\text { Hea }}$ $\begin{array}{lllllll}.01 & .05 & .17 & .28 & .02 & .07 & \text { End }\end{array}$ 
$\underline{\text { Gender and Occupational Class Process }}$

$\underline{\text { Selector }}$

$\begin{array}{lllllllll}\frac{\mathrm{T}^{0}}{\text { Com }} & \underline{\mathrm{P}} & \frac{\mathrm{T}^{1}}{\mathrm{M}} & \frac{\mathrm{pf}}{\mathrm{M}} & \frac{\mathrm{pip}}{\mathrm{puf}} & \underline{\mathrm{pf}} & \text { pip } & \underline{\mathrm{puf}}\end{array}$ $\begin{array}{lllllllll}\underline{\mathrm{Edu}} & .23 & \underline{\mathrm{W}} & 1 . & 0 & 0 & 0 & 0 & 0\end{array}$ $\begin{array}{lllllllll}\underline{\text { Tra }} & .05 & \underline{\text { Lea }} & 0 & 1 . & 0 & 0 & 0 & 0\end{array}$ $\begin{array}{lllllllll}\underline{\text { Pol }} & .30 & \underline{\text { Exp }} & 0 & 0 & 0 & 0 & 1 . & 0\end{array}$

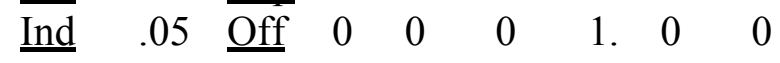
$\begin{array}{lllllllll}\underline{\mathrm{Hea}} & .25 & \underline{\mathrm{Ser}} & 0 & 0 & 0 & 0 & .49 & .51\end{array}$

$\underline{\mathrm{T}^{1}} \quad \underline{\mathrm{P}} \quad \underline{\mathrm{T}^{2}} \quad \underline{\mathrm{pf}} \quad \underline{\text { pip }} \quad \underline{\text { puf }} \quad \underline{\text { pf }}$ pip $\begin{array}{lllllllll}\text { Com } & .23 & \underline{\mathrm{M}} & 0 & 1 . & 0 & 0 & 0 & 0\end{array}$ $\begin{array}{lllllllll}\underline{\mathrm{Edu}} & .05 & \underline{\mathrm{W}} & 0 & 0 & 1 . & 0 & 0 & 0\end{array}$

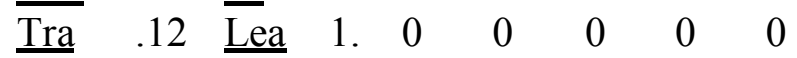

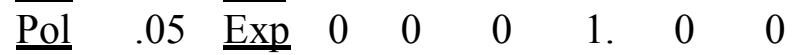
$\begin{array}{lllllllll}\underline{\text { Ind }} & .42 & \underline{\text { Off }} & 0 & 0 & 0 & 0 & 1 . & .31\end{array}$ $\begin{array}{lllllllll}\underline{\mathrm{Hea}} & .13 & \underline{\mathrm{Ser}} & 0 & 0 & 0 & .49 & .25 & .26\end{array}$

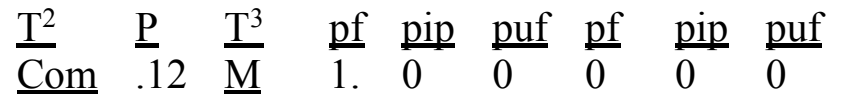
$\begin{array}{lllllllll}\underline{\mathrm{Edu}} & .23 & \underline{\mathrm{W}} & 0 & 1 . & 0 & 0 & 0 & 0\end{array}$ $\begin{array}{lllllllll}\text { Tra } & .05 & \underline{\text { Lea }} & 0 & 0 & 1 . & 0 & 0 & 0\end{array}$

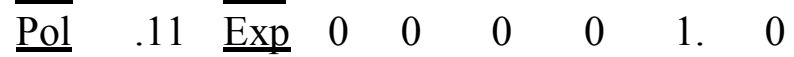
$\begin{array}{lllllllll}\underline{\text { Ind }} & .45 & \underline{\text { Off }} & 0 & 0 & 0 & 1 . & 0 & .31\end{array}$ $\begin{array}{lllllllll}\underline{\mathrm{Hea}} & .03 & \underline{\mathrm{Ser}} & 0 & 0 & 0 & .25 & .62 & .13\end{array}$

$\begin{array}{lllllllll}\frac{\mathrm{T}^{3}}{\mathrm{Com}} & \underline{\mathrm{P}} & \frac{\mathrm{T}^{4}}{12} & \underline{\mathrm{pf}} & \underline{\mathrm{p}} \mathrm{p} & \underline{\mathrm{puf}} & \underline{\mathrm{pf}} & \underline{\mathrm{pip}} & \underline{\text { puf }}\end{array}$

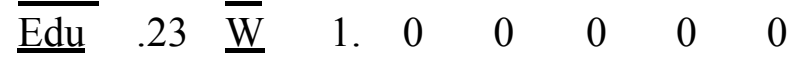

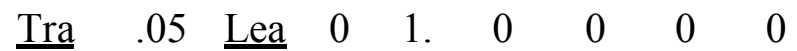

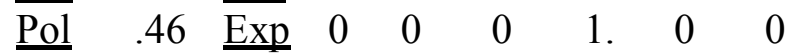

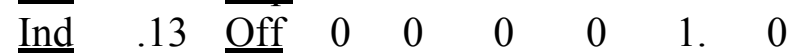
$\begin{array}{lllllllll}\underline{\underline{H e a}} & 0 & \underline{\mathrm{Ser}} & 0 & 0 & 0 & .62 & .32 & .07\end{array}$

$\begin{array}{lllllllll}\frac{\mathrm{T}^{4}}{\mathrm{Com}} & \underline{\mathrm{P}} & \frac{\mathrm{T}^{5}}{23} & \underline{\mathrm{pf}} & \underline{\mathrm{p}} \mathrm{p} & \underline{\mathrm{puf}} & \underline{\mathrm{pf}} & \underline{\mathrm{pip}} & \underline{\mathrm{puf}}\end{array}$ $\begin{array}{lllllllll}\underline{\mathrm{Edu}} & .05 & \underline{\mathrm{W}} & 0 & 0 & 1 . & 0 & 0 & 0\end{array}$

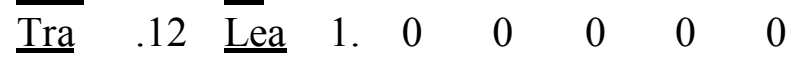
$\begin{array}{lllllllll}\underline{\text { Pol }} & .47 & \underline{\text { Exp }} & 0 & 0 & 0 & 0 & 1 . & 0\end{array}$

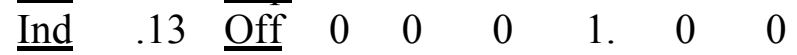
$\begin{array}{lllllllll}\underline{\mathrm{Hea}} & 0 & \underline{\mathrm{Ser}} & 0 & 0 & 0 & .32 & .65 & .04\end{array}$
$\begin{array}{lllllllll}\mathrm{T}^{5} & \underline{\mathrm{P}} & \underline{\mathrm{T}^{6}} & \underline{\mathrm{pf}} & \underline{\text { pip }} & \underline{\mathrm{puf}} & \underline{\mathrm{pf}} & \underline{\mathrm{pip}} & \text { puf }\end{array}$ $\begin{array}{lllllllll}\underline{\mathrm{Edu}} & .23 & \underline{\mathrm{W}} & 0 & 1 . & 0 & 0 & 0 & 0\end{array}$

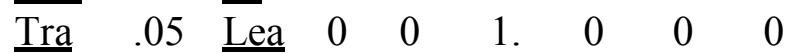

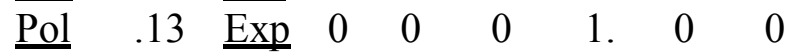

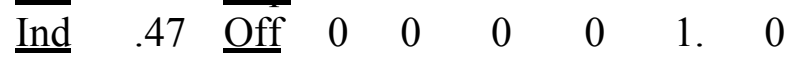
$\begin{array}{lllllllll}\underline{\mathrm{Hea}} & 0 & \underline{\mathrm{Ser}} & 0 & 0 & 0 & .65 & .33 & .02\end{array}$

\section{Opter}

$\begin{array}{lllllllll}\mathrm{T}^{0} & \underline{\mathrm{P}} & \frac{\mathrm{T}^{1}}{\mathrm{Com}} & \underline{\mathrm{cla}} & \underline{\mathrm{chu}} & \underline{\mathrm{ord}} & \underline{\text { ass }} & \underline{\bmod } & \underline{\mathrm{tra}}\end{array}$ $\begin{array}{lllllllll}\underline{\mathrm{Edu}} & .23 & \underline{\mathrm{W}} & 0 & 0 & 0 & 0 & 0 & 0\end{array}$

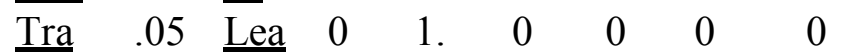
$\begin{array}{lllllllll}\underline{\mathrm{Pol}} & .13 & \underline{\mathrm{Exp}} & 0 & .73 & 0 & .27 & 0 & 0\end{array}$ $\begin{array}{lllllllll}\underline{\text { Ind }} & .47 & \underline{\text { Off }} & 0 & .34 & .66 & 0 & 0 & 0\end{array}$ $\begin{array}{lllllllll}\underline{\mathrm{Hea}} & 0 & \underline{\mathrm{Ser}} & 0 & 0 & .30 & 0 & .27 & .43\end{array}$

$\begin{array}{lllllllll}\underline{\mathrm{T}}^{1} & \underline{\mathrm{P}} & \underline{\mathrm{T}^{2}} & \underline{\mathrm{cla}} & \underline{\mathrm{chu}} & \frac{\text { ord }}{\mathrm{Com}} & \underline{\text { ass }} & \underline{\bmod } & \underline{\mathrm{tra}}\end{array}$ $\begin{array}{lllllllll}\underline{\underline{\mathrm{Edu}}} & .31 & \underline{\mathrm{W}} & 0 & 0 & 0 & 0 & 0 & 0\end{array}$ $\begin{array}{lllllllll}\text { Tra } & .30 & \underline{\text { Lea }} & 0 & 0 & 0 & 0 & 0 & 0\end{array}$ $\begin{array}{lllllllll}\underline{\underline{P o l}} & .04 & \underline{\mathrm{Exp}} & 0 & .20 & 0 & .07 & 0 & 0\end{array}$ $\begin{array}{lllllllll}\underline{\text { Ind }} & 0 & \underline{\text { Off }} & 0 & .66 & 0 & 1 . & 0 & 0\end{array}$ $\begin{array}{lllllllll}\underline{\text { Hea }} & 0 & \underline{\text { Ser }} & 0 & .39 & .31 & 0 & .12 & .18\end{array}$

$\begin{array}{lllllllll}\underline{\mathrm{T}}^{2} & \underline{\mathrm{P}} & \underline{\mathrm{T}^{3}} & \underline{\mathrm{cla}} & \underline{\mathrm{chu}} & \underline{\mathrm{ord}} & \underline{\text { ass }} & \frac{\bmod }{0} & \frac{\text { tra }}{0}\end{array}$ $\begin{array}{lllllllll}\underline{\mathrm{Edu}} & .01 & \underline{\mathrm{W}} & 0 & 0 & 0 & 0 & 0 & 0\end{array}$ $\begin{array}{lllllllll}\underline{\text { Tra }} & 0 & \underline{\text { Lea }} & 0 & 0 & 0 & 0 & 0 & 0\end{array}$

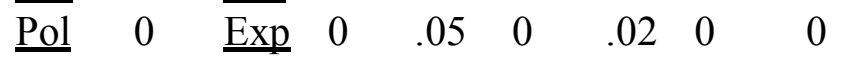

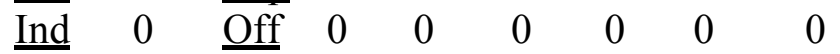
$\begin{array}{lllllllll}\underline{\mathrm{Hea}} & 0 & \underline{\mathrm{Ser}} & 0 & .34 & .13 & 0 & .05 & .08\end{array}$ 


\section{Chooser}

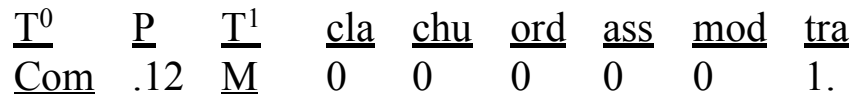
$\begin{array}{lllllllll}\underline{\mathrm{Edu}} & 0 & \underline{\mathrm{W}} & 0 & 0 & 0 & 0 & 1 . & 0\end{array}$ $\begin{array}{lllllllll}\text { Tra } & 0 & \underline{\text { Lea }} & 0 & 0 & 0 & 0 & 0 & 0\end{array}$ $\begin{array}{lllllllll}\underline{\mathrm{Pol}} & 0 & \underline{\mathrm{Exp}} & 0 & 0 & .61 & .39 & 0 & 0\end{array}$ $\underline{\underline{\text { Ind }}} \quad 0 \quad \begin{array}{lllllll}\text { Off } & 0 & 0 & 1 . & 0 & 0 & 0\end{array}$ $\begin{array}{lllllllll}\underline{\text { Hea }} & 0 & \underline{\underline{\text { Ser }}} & 1 . & 0 & 0 & 0 & 0 & 0\end{array}$

$\begin{array}{lllllllll}\mathrm{T}^{1} & \underline{\mathrm{P}} & \frac{\mathrm{T}^{2}}{\mathrm{Com}} & \frac{\text { cla }}{\mathrm{M}} & \frac{\text { chu }}{0} & \frac{\text { ord }}{0} & \frac{\text { ass }}{0} & \frac{\bmod }{0} & \frac{\text { tra }}{0}\end{array}$ $\begin{array}{lllllllll}\underline{\mathrm{Edu}} & 0 & \underline{\mathrm{W}} & 0 & 0 & 1 . & 0 & 0 & 0\end{array}$ $\begin{array}{lllllllll}\text { Tra } & 0 & \text { Lea } & 0 & 0 & 0 & 0 & 0 & 0\end{array}$

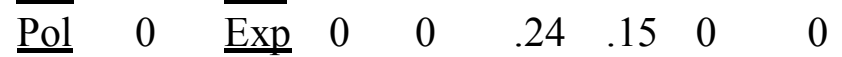
$\begin{array}{lllllllll}\underline{\text { Ind }} & 0 & \underline{\text { Off }} & 0 & 0 & 0 & 0 & 0 & 0\end{array}$ $\begin{array}{lllllllll}\underline{\text { Hea }} & .12 & \underline{\text { Ser }} & 0 & 0 & 0 & 0 & 0 & 1 .\end{array}$

$\begin{array}{llllll}\text { Com } & \underline{\text { Edu }} & \frac{\text { Tra }}{0} & \frac{\text { Pol }}{0} & \underline{\text { Ind }} & \underline{\text { Hea }}\end{array}$

Environments Process

$\underline{\text { Selector }}$

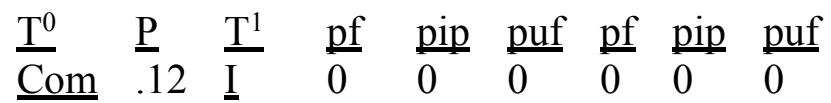
$\begin{array}{lllllllll}\mathrm{Edu} & .23 & \mathrm{Pg} & 0 & 1 . & 0 & 0 & 0 & 0\end{array}$ $\begin{array}{lllllllll}\underline{\mathrm{Tra}} & .05 & \overrightarrow{\mathrm{Sg}} & .62 & 0 & .38 & 0 & 0 & 0\end{array}$ $\begin{array}{lllllllll}\underline{\mathrm{Pol}} & .30 & \underline{\mathrm{Pag}} & 0 & 0 & 0 & 0 & 0 & 1 .\end{array}$ $\begin{array}{lllllllll}\text { Ind } & .05 & \overline{\mathrm{Org}} & 0 & 0 & 0 & 0 & 1 . & 0\end{array}$ $\begin{array}{lllllllll}\underline{\text { Hea }} & .25 & \underline{\text { Pop }} & 0 & 0 & 0 & 1 . & 0 & 0\end{array}$

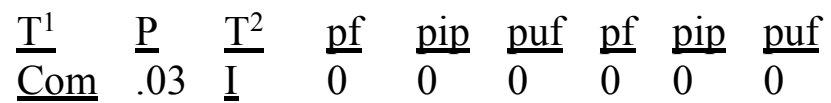
$\begin{array}{lllllllll}\underline{\mathrm{Edu}} & .23 & \mathrm{Pg} & 0 & 1 . & 0 & 0 & 0 & 0\end{array}$ $\begin{array}{lllllllll}\mathrm{Tra} & .02 & \overrightarrow{\mathrm{Sg}} & .24 & 0 & .14 & 0 & 0 & 0\end{array}$ $\begin{array}{lllllllll}\underline{\mathrm{Pol}} & .25 & \mathrm{Pag} & 0 & 0 & 0 & 1 . & 0 & 0\end{array}$ $\begin{array}{lllllllll}\text { Ind } & .05 & \text { Org } & 0 & 0 & 0 & 0 & 1 . & 0\end{array}$ $\begin{array}{lllllllll}\underline{\text { Hea }} & .30 & \underline{\text { Pop }} & 0 & 0 & 0 & 0 & 0 & 1 .\end{array}$

\section{$\underline{\text { Opter }}$}

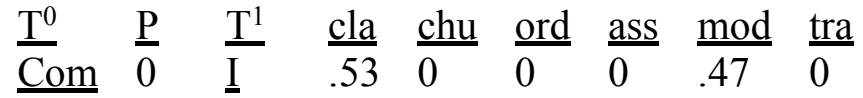
$\begin{array}{lllllllll}\mathrm{Edu} & .23 & \underline{\mathrm{Pg}} & .20 & 0 & 0 & 0 & 0 & .80\end{array}$ $\begin{array}{lllllllll}\mathrm{Tra} & 0 & \overrightarrow{\mathrm{Sg}} & 0 & 1 . & 0 & 0 & 0 & 0\end{array}$ $\begin{array}{lllllllll}\underline{\mathrm{Pol}} & .25 & \underline{\mathrm{Pag}} & 0 & 0 & .79 & 0 & .21 & 0\end{array}$ $\begin{array}{lllllllll}\text { Ind } & .05 & \underline{\text { Org }} & 0 & .43 & .40 & .17 & 0 & 0\end{array}$ $\begin{array}{lllllllll}\text { Hea } & .30 & \underline{\text { Pop }} & 0 & 0 & 0 & 0 & .42 & .58\end{array}$

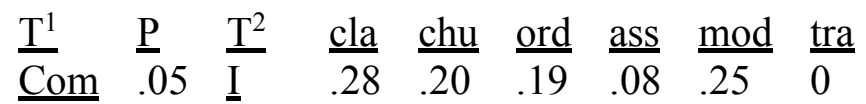
$\begin{array}{lllllllll}\underline{\mathrm{Edu}} & .02 & \mathrm{Pg} & .10 & 0 & 0 & 0 & .43 & .46\end{array}$ $\begin{array}{lllllllll}\underline{\mathrm{Tra}} & .22 & \underline{\mathrm{Sg}} & .20 & 0 & 0 & 0 & 0 & .80\end{array}$ $\begin{array}{lllllllll}\underline{\mathrm{Pol}} & .01 & \underline{\mathrm{Pag}} & 0 & .88 & .08 & .04 & 0 & 0\end{array}$

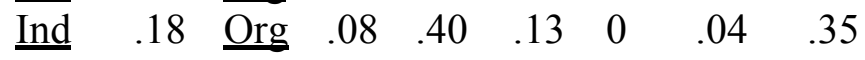
$\begin{array}{lllllllll}\underline{\text { Hea }} & .36 & \underline{\underline{\text { Pop }}} & 0 & .18 & .17 & .07 & .24 & .33\end{array}$

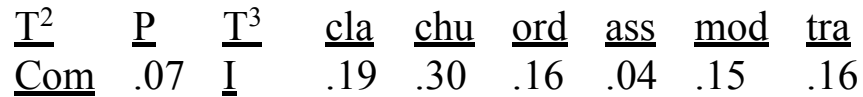
$\begin{array}{lllllllll}\underline{\mathrm{Edu}} & .16 & \mathrm{Pg} & .06 & .19 & .17 & .07 & .24 & .27\end{array}$ $\begin{array}{lllllllll}\mathrm{Tra} & .09 & \mathrm{Sg} & .10 & 0 & 0 & 0 & .43 & .46\end{array}$ $\begin{array}{lllllllll}\underline{\mathrm{Pol}} & .03 & \underline{\mathrm{Pag}} & .17 & .09 & .03 & 0 & .01 & .70\end{array}$ $\begin{array}{lllllllll}\text { Ind } & .12 & \underline{\operatorname{Org}} & .12 & .15 & .01 & .01 & .19 & .52\end{array}$ $\begin{array}{lllllllll}\underline{\text { Hea }} & .37 & \underline{\underline{P o p}} & .04 & .27 & .15 & .04 & .16 & .34\end{array}$

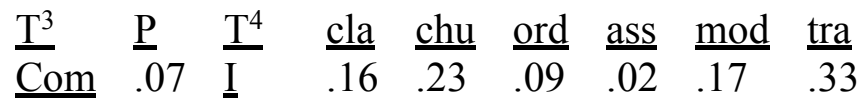
$\begin{array}{lllllllll}\mathrm{Edu} & .17 & \underline{\mathrm{Pg}} & .07 & .28 & .16 & .04 & .15 & .30\end{array}$ $\begin{array}{lllllllll}\text { Tra } & .10 & \underline{\mathrm{Sg}} & .05 & .19 & .17 & .07 & .25 & .27\end{array}$ $\begin{array}{lllllllll}\underline{\mathrm{Pol}} & .03 & \underline{\mathrm{Pag}} & .11 & .03 & 0 & 0 & .38 & .47\end{array}$

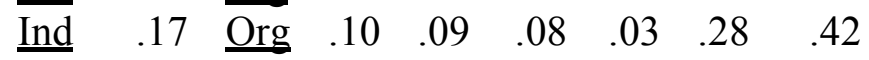
$\begin{array}{lllllllll}\underline{\text { Hea }} & .30 & \underline{\underline{P o p}} & .07 & .22 & .10 & .03 & .17 & .41\end{array}$

\section{$\underline{\text { Chooser }}$}

$\begin{array}{lllllllll}\mathrm{T}^{0} & \underline{\mathrm{P}} & \frac{\mathrm{T}}{\mathrm{Com}} & \frac{\mathrm{cre}}{\mathrm{I}} & \frac{\text { bel }}{0} & \underline{\mathrm{vie}} & \underline{\mathrm{inf}} & \frac{\mathrm{kno}}{0} & \frac{\mathrm{knh}}{.89}\end{array}$ $\begin{array}{lllllllll}\underline{\mathrm{Edu}} & .16 & \underline{\mathrm{Pg}} & 0 & 0 & 0 & 0 & 1 . & 0\end{array}$ $\begin{array}{lllllllll}\text { Tra } & .09 & \underline{\mathrm{Sg}} & .15 & 0 & 0 & .85 & 0 & 0\end{array}$ $\begin{array}{lllllllll}\underline{\underline{P o l}} & .03 & \underline{\mathrm{Pag}} & .80 & 0 & 0 & .20 & 0 & 0\end{array}$ $\begin{array}{lllllllll}\text { Ind } & .17 & \text { Org } & 0 & 0 & 1 . & 0 & 0 & 0\end{array}$ $\begin{array}{lllllllll}\underline{\text { Hea }} & .31 & \underline{\underline{P o p}} & 0 & .43 & .57 & 0 & 0 & 0\end{array}$ 
$\begin{array}{lllllllll}\mathrm{T}^{1} & \underline{\mathrm{P}} & \underline{\mathrm{T}^{2}} & \underline{\mathrm{cre}} & \underline{\text { bel }} & \frac{\mathrm{vie}}{\mathrm{Com}} & \underline{\mathrm{inf}} & \underline{\mathrm{kno}} & \underline{\mathrm{knh}}\end{array}$

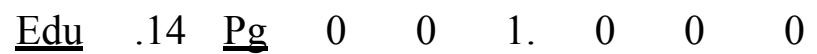

$\begin{array}{lllllllll}\underline{\mathrm{Tra}} & .35 & \underline{\mathrm{Sg}} & .68 & .02 & 0 & .17 & 0 & .13\end{array}$

$\begin{array}{lllllllll}\underline{\mathrm{Pol}} & .08 & \underline{\mathrm{Pag}} & .16 & .09 & 0 & .04 & 0 & .71\end{array}$

$\begin{array}{lllllllll}\underline{\text { Ind }} & .16 & \underline{\mathrm{Org}} & .15 & 0 & 0 & .85 & 0 & 0\end{array}$

$\begin{array}{lllllllll}\underline{\text { Hea }} & .06 & \underline{\text { Pop }} & .09 & 0 & 0 & .48 & .43 & 0\end{array}$

$\begin{array}{lllllllll}\frac{\mathrm{T}^{2}}{\text { Com }} & \frac{\mathrm{P}}{.28} & \frac{\mathrm{T}^{3}}{\mathrm{I}} & \frac{\text { cre }}{.08} & \frac{\text { bel }}{0} & \frac{\text { vie }}{.11} & \frac{\text { inf }}{.43} & \frac{\mathrm{kno}}{.38} & \frac{\mathrm{knh}}{0}\end{array}$

$\begin{array}{lllllllll}\underline{\mathrm{Edu}} & .03 & \underline{\mathrm{Pg}} & .15 & 0 & 0 & .85 & 0 & 0\end{array}$

$\begin{array}{lllllllll}\underline{\mathrm{Tra}} & 16 & \underline{\mathrm{Sg}} & .14 & .13 & .08 & .03 & .02 & .60\end{array}$

$\begin{array}{lllllllll}\underline{\mathrm{Pol}} & .23 & \underline{\mathrm{Pag}} & .03 & .32 & .41 & .01 & .09 & .14\end{array}$

$\begin{array}{lllllllll}\underline{\text { Ind }} & .03 & \underline{\mathrm{Org}} & .68 & .02 & 0 & .17 & 0 & .13\end{array}$

$\begin{array}{lllllllll}\underline{\text { Hea }} & .11 & \underline{\text { Pop }} & .39 & 0 & .43 & .10 & 0 & .08\end{array}$

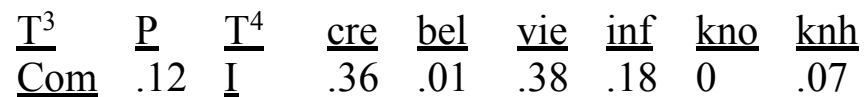
$\begin{array}{lllllllll}\underline{\mathrm{Edu}} & .10 & \underline{\mathrm{Pg}} & .68 & .02 & 0 & .17 & 0 & .13\end{array}$ $\begin{array}{lllllllll}\text { Tra } & .18 & \underline{\mathrm{Sg}} & .04 & .28 & .36 & .07 & .13 & .12\end{array}$ $\begin{array}{lllllllll}\underline{\mathrm{Pol}} & .17 & \underline{\mathrm{Pag}} & .07 & .06 & .17 & .35 & .32 & .03\end{array}$ $\begin{array}{lllllllll}\underline{\text { Ind }} & .13 & \underline{\mathrm{Org}} & .14 & .13 & .08 & .03 & .02 & .60\end{array}$ $\begin{array}{lllllllll}\underline{\text { Hea }} & .14 & \underline{\text { Pop }} & .14 & .08 & .04 & .38 & .01 & .34\end{array}$

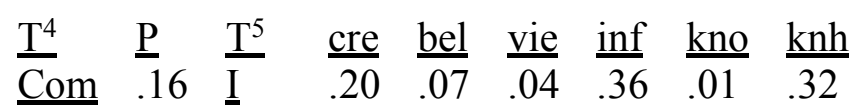

$\begin{array}{lllllllll}\underline{\mathrm{Edu}} & .09 & \underline{\mathrm{Pg}} & .14 & .13 & .08 & .03 & .02 & .60\end{array}$

$\begin{array}{lllllllll}\frac{\mathrm{Tra}}{\mathrm{Pa}} & .15 & \underline{\mathrm{Sg}} & .11 & .06 & .20 & .32 & .28 & .03\end{array}$

$\begin{array}{lllllllll}\underline{\mathrm{Pol}} & .17 & \underline{\mathrm{Pag}} & .30 & .02 & .34 & .21 & .06 & .06\end{array}$

$\begin{array}{lllllllll}\underline{\text { Ind }} & .08 & \underline{\mathrm{Org}} & .04 & .28 & .36 & .07 & .13 & .12\end{array}$

$\begin{array}{lllllllll}\underline{\text { Hea }} & .17 & \underline{\text { Pop }} & .31 & .16 & .21 & .11 & .08 & .13\end{array}$

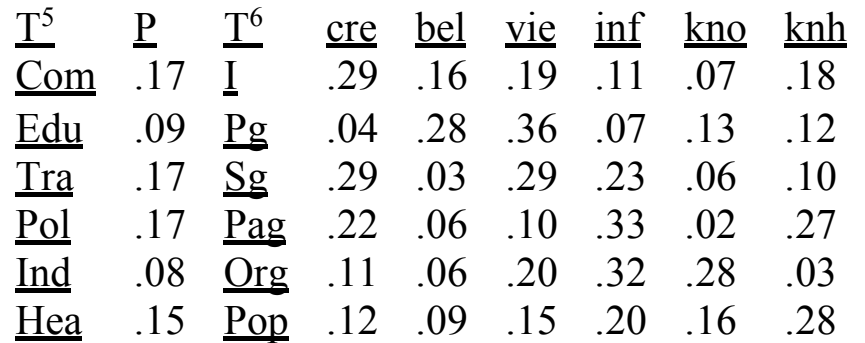

$\begin{array}{lllllll}\frac{\text { Com }}{.17} & \frac{\text { Edu }}{.08} & \frac{\text { Tra }}{.17} & \frac{\text { Pol }}{.18} & \underline{\text { Ind }} & \underline{\text { Hea }} & \\ .08 & \frac{.15}{\text { End }}\end{array}$

Note. The ones in the tables are calculative, only.

\section{Discussion}

The hypothesis stated that gender, socioeconomic status, occupational class, and social environments regulate action of the mind processes Selector, Opter, and Chooser. The. hypothesis corroborates. The background processes do not follow up with the process without the background variables, and the background processes differ from each other. Furthermore, the background processes include in their specific effects. As to the use of the percepts there is a ground. Hardly, a person has an organized conception of the whole industry of a country, for example. Therefore, comprehensions base on varying perceptual processing, which results in the percepts. The question is about defective information.

At the start of Selector of the gender and socioeconomic group process, the fact that the participants are women brings them forth to finish the educational percepts. Next, they move to the commercial percepts. 
The fact that the participants are the upper level employees generates them to be getting the political percepts ready. The fact that the participants are the manual workers generates them to be making the health care percepts ready. The political, and health care percepts remain half-finished. The upper level employees, and the manual workers transfer to the industrial percepts. During the first loop, to be a lower level employee engenders the participants to be making the industrial percepts ready, and they adhere to the industrial percepts. The industrial percepts remain half-finished. In the second loop, the fact that the participants are the lower level employees causes them to finish the industrial percepts. Thereafter, they switch to the political percepts. In the third loop, to be an upper level employee causes the participants to finish the political percepts. The fact that the participants are the manual workers makes them to finish the health care percepts. The upper level employees stick to the political percepts while the manual workers switch from the health care percepts to the political percepts before Opter.

In the beginning of Opter, the fact that the participants are the upper level employees causes them to sort the political percepts into heaps, and to arrange them properly. After that, they switch to the educational, and traffic percepts. During the first loop, female sex causes the participants to change the educational percepts into the health care percepts. Being a self-employed person, makes the participants to convert the traffic percepts into the health care percepts. Women, and the self-employed persons stick to the health care percepts. In the second loop, to be a manual worker makes the participants to change the health care percepts into different health care percepts. The manual workers adhere to the health care percepts. During the third loop, the fact that the participants are the manual workers causes them to change the health care percepts into other health care percepts, and they continue with the health care percepts. 
In the fourth loop, the fact that the participants are women occasions them to turn the health care percepts into different health care percepts. The fact that the participants are the manual workers effects them to convert the health care percepts into different health care percepts. Women, and the manual workers stick to the health care percepts before Chooser.

At the start of Chooser, the fact that the participants are women engenders them to tell how healthcare functions in reality, and they adhere to the health care percepts. The fact that the participants are the self-employed persons occasions them to give knowledge of traffic facts. Thereafter, they transfer to the political percepts. The fact that the participants are the manual workers causes them to assume healthcare. After that they move to the educational percepts. In the first loop, the fact that the participants are the upper level employees makes them to give knowledge of political facts, and they adhere to the political percepts. The fact that the participants are the manual workers occasions them to tell how healthcare operates in reality and they stick to the health care percepts. In the second loop, the fact that the participants are the upper level employees occasions them to express their personal comprehensions about politics. Thereafter, they shift to the traffic percepts. In the third loop, the fact that the participants are the self-employed persons occasions them to express their personal comprehensions about traffic, and they adhere to the traffic percepts. In the fourth loop, to be a self-employed person causes the participants to give knowledge of traffic facts. Next, they shift to the political percepts. In the fifth loop, the fact that the participants are the self-employed persons causes them to express personal comprehensions about traffic, and they adhere to the traffic percepts. The fact that the participants are the upper level employees causes them to give knowledge of political facts, and they stick to the political percepts. The traffic, and the political percepts remain the upshots. What is remarkable at this point is the process does not reach equilibrium but it continues traffic, traffic, politics, and politics. 
In the beginning of Selector of the gender and occupational class process, female sex generates the participants to finish the educational percepts, and after that they transfer to the commercial percepts. The fact that the participants are the experts causes them to be getting the political percepts ready. Thereafter, they switch to the industrial percepts. The political percepts remain half-finished. During the first loop, the fact that the participants are men occasions them to leave the commercial percepts half-finished. Thereafter, they switch to the educational percepts. The fact that the participants are the officers causes them to be making the industrial percepts ready, and they stick to the industrial percepts. During the second loop, the fact that the participants are women causes them to be getting the educational percepts ready, and they adhere to the educational percepts. The educational percepts remain halffinished. To be an officer effects the participants to finish the industrial percepts. Thereafter, they switch to the political percepts. In the third loop, the fact that the participants are women makes them to finish the educational percepts. After that, they move to the commercial percepts. The fact that the participants are the experts effects them to finish the political percepts, and they continue with the political percepts. In the fourth loop, the fact that the participants are men engenders them to be making the commercial percepts ready. Next, they shift to the educational percepts. The commercial percepts remain half-finished. To be an expert occasions the participants to be getting the political percepts ready. Thereafter, they switch to the industrial percepts. The political percepts of the experts remain half-finished. During the fifth loop, being a woman causes the participants to be making the educational percepts ready, and they adhere to them. The educational percepts of women remain halffinished. The fact that the participants are the officers, occasions them to be making the industrial percepts ready, and they adhere to the industrial percepts before Opter. The industrial percepts remain half-finished. 
At the start of Opter, the fact that the participants are the officers effects them to sort the industrial percepts into heaps, and to arrange them properly. Thereafter, they move to the educational and traffic percepts. During the first loop, the fact that the participants are men, causes them to arrange the commercial percepts properly, and they stick to the commercial percepts. In the second loop, the fact that the participants are men causes them to put the commercial percepts into proper categories, and to stick to them before Chooser.

In the beginning of Chooser, the fact that the participants are men occasions them to tell how commerce operates in reality. Thereafter, they shift to the health care percepts. During the first loop, the fact that the participants are the servants occasions them to tell how healthcare functions in reality, and the health care percepts remain the upshot.

In the beginning of Selector of the social environments process, the primary group as the referent brings forth the participants (the participants, who refer) to be getting the educational percepts ready, and to continue with them. The educational percepts remain halffinished. The political activity group as the referent generates the participants not to finish the political percepts, and they switch to the health care percepts. The political percepts remain unfinished. The population as the referent brings forth the participants to finish the health care percepts, and they shift to the political percepts. During the only loop before Opter, the primary group as the referent causes the participants to be getting the educational percepts ready, and to stick to them. The educational percepts remain half-finished. The political activity group as the referent occasions the participants to finish the political percepts, and to adhere to them. The population as the referent causes the participants not to finish the health care percepts but they stick to them. The healthcare percepts remain unfinished.

At the start of Opter, the primary group as the referent engenders the participants to change the educational percepts into the healthcare percepts. The political activity group as 
the referent engenders the participants to arrange the political percepts properly. Thereafter, they move to the traffic percepts. The population as the referent occasions the participants to convert the health care percepts into different health care percepts. In the first loop, the secondary group as the referent makes the participants to convert the health care percepts into other health care percepts. In the second loop, the population as the referent occasions the participants to turn the health care percepts into different health care percepts. During the third loop, the population as the referent makes the participants to change the health care percepts into different health care percepts before Chooser.

At the start of Chooser, the organization, and the population as the referents cause the participants to express personal comprehensions about industry, and healthcare. Thereafter, the participants shift to the traffic percepts. During the first loop, the secondary group as the referent occasions the participants to rely on their sources of traffic information. After that, the participants switch to the commercial percepts. The organization as the referent causes the participants to give knowledge of industrial facts. Thereafter, they switch to the political percepts. In the second loop, the individual as the referent occasions the participants to give knowledge of commercial facts. Thereafter they shift to the political percepts. The political activity group as the referent causes the participants to express personal comprehensions about politics. Thereafter, the participants move to the traffic percepts. During the third loop, the political activity group as the referent occasions the participants to give knowledge of political facts, and they stick to the political percepts. The population as the referent causes the participants to give knowledge of health care facts. Thereafter, they switch to the political percepts. The organization as the referent causes the participants to tell how industry works in reality. After that, they move to the health care percepts. During the fourth loop, the political activity group, and the population as the referents occasion the participants to rely on their 
sources of political, and health care information. Thereafter, they move to the commercial percepts. The political activity group as the referent causes the participants to express personal comprehensions about politics. After that, they transfer to the traffic percepts. The organization as the referent engenders the participants to express personal comprehensions about industry. After that, they move to the traffic percepts. The individual, and the secondary group as the referents cause the participants to give knowledge of commercial, and traffic facts. Thereafter, they switch to the political percepts. In the last loop, the political activity group as the referent makes the participants to give knowledge of political facts, and the political percepts remain the upshot. Summarily, it is verifiable from the previous.

In the gender and socioeconomic group process, during Selector being a woman, an upper level employee, a lower level employee or a manual worker makes the participants to finish the conscious, experiential, educational, political, industrial, and health care processes. The conscious, experiential, political processes remain valid before Opter. During Opter, being a woman, a self-employed person or a manual worker causes the participants to change the conscious, experiential, educational, health care, and traffic processes into other processes. Being an upper level employee is an exception, which makes the participants to sort the conscious, experiential, political processes in heaps, and to arrange the processes properly. The transfers take place to the health care percepts with the exception of the upper level employees, who transfer to the educational, and traffic percepts before Chooser. During Chooser, female sex brings about the participants to tell how health care works in reality. To be a self-employed person or an upper level employee causes the participants to give knowledge of political, and traffic facts, and to express personal comprehensions about 
politics, and traffic. The fact that the participants are the manual workers engenders them s to assume, and to tell how health care things work in reality.

In the gender and occupational class process, during Selector being a man, a woman, an expert or an officer causes the participants to remain in half-finished in construction of the conscious, experiential, commercial, educational, political, and industrial processes. Although, they finish other corresponding processes. The educational, and industrial processes continue into Opter. During Opter male sex occasions the participants to put the conscious, experiential, commercial processes into categories, and to arrange the processes properly. Being an officer brings about the participants to sort the conscious, experiential, industrial processes in heaps, and arrange the processes properly. The commercial processes continue into Chooser. During Chooser, male sex engenders the participants to tell how commercial things function in reality. Being a servant effects the participants to tell how health care things operate in reality.

In the environments process, during Selector the referent of the primary group causes the participants to be preparing the conscious, experiential, educational processes The referent of the political activity group causes the participants to finish the conscious, experiential, political processes. The referent of the population brings forth the participants not to finish the conscious, experiential, health care processes. Processing of the educational, political, and heath care percepts continue in Opter. In Opter, the referent of the primary group causes the participants to convert the conscious, experiential, educational processes into the health care processes. The referent of the secondary group, and of the population cause the participants to convert the conscious, experiential, health care processes into different health care processes. The referent of the political activity group engenders the participants to arrange the conscious, experiential, 
political processes properly. The health care processes continue into Chooser. During Chooser, the referent of the individual causes the participants to give knowledge of the commercial facts. the referent of the secondary group engenders the participants to rely on their sources of traffic information, and to give knowledge of traffic facts. The referent of the political activity group occasions the participants to trust in their sources of political information, to give knowledge of political facts, and to express personal comprehensions about politics. The referent of the organization makes the participants to express personal comprehensions about industry, to give knowledge of industrial facts, and to tell how industrial things work in reality. The referent of the population makes the participants to rely on their sources of health care information, to express personal comprehensions about healthcare, and to give knowledge of health care facts.

In a theoretical sight, the scrutinized system can be characterized as a consequent, causal system where the causes do not occur continuously but at different times in the processes. Removal of repetitions (redundancy) reveals that causal mappings occur between the background variables, and behavior. Female sex, the upper level employee, the lower level employee, and the manual worker cause to finish the conscious, experiential processes, and thereafter the transitions to other processes, principally. Female sex, the self-employed person, and the manual worker cause to change the processes in the different processes. The upper level employee causes to sort the conscious experiential processes in heaps, and to arrange them properly, and not to move to other processes., mainly. Female sex, and the manual worker cause to tell how things work in reality. In addition, the manual worker causes to assume. The self-employed person, and the upper level employee cause to express personal comprehensions, and to tell facts without moving to other processes, mainly. So, there are 
many to one, one to many, and one to one mapping in the gender and socioeconomic group process. In the gender and occupational class process, male sex, female sex, the expert, and the officer cause to leave the conscious, experiential processes half-finished, and move to other processes, chiefly. Male sex, and the officer cause to sort in categories, and properly with the transitions, and no transitions. Male sex, and the servant cause to tell how things operate in reality with one transition, and adherence. Thus, there are many to one, one to many, and one to one mapping. During the environments process, the primary group causes to leave the conscious, experiential processes half-finished. The political activity group, and the population cause to finish, and not to finish the processes with no transitions, mostly. The primary group, the secondary group, and the population cause to change the conscious, experiential processes into the different processes, The political activity group causes to arrange the processes properly with remaining in the processes, chiefly. The individual causes to tell facts. The secondary group causes to rely on the sources of information, and to tell facts The political activity group, and the population cause to trust in the sources of information, to present personal comprehensions, and to tell facts. The organization causes to present personal comprehensions, to tell facts, and to express how things function in reality with the transitions to other processes, in most cases.

As to the model mentioned in introduction one has to verify that the former model includes in the current process model but only conceptually, and theoretically because the current research bases on spoken language. A fact that simultaneously is a defectiveness because action, and task performance lack. From the viewpoint of information processing one is able to state that the form bound contents or the information entities remain defective because of a shortage of familiarity with intensions in societal life. 


\section{References}

Bertalanffy, L. (1968). General system theory. (Third Printing). New York, NY: George Braziller, Inc.

Bickhard, M. H. (2003). Mind as process. Retrieved December 22, 2017, from http://www.lehigh.edu/ mhb0/mhb0.html

Freyd, J. J. !987). Dynamic mental representations. Psychotically Review, Vol 94, No. 4, 427-438. Retrieved December 24, 2017 from http://wexler.free.fr/library/files/freyd $\% 20 \% 281987 \% 29 \% 20$ dynamic $\% 20$ mental $\% 20$ representations.

Fusella, P. V. (2013). Dynamic systems theory in cognitive science: Major elements, applications, and debates surrounding a revolutionary meta-theory. Dynamical Psychology 2013. Retrieved December 24, 2017 from http://dynapsyc.org/2013/Fusella.

Laasonen, R. J. (2007). If the mind then behavior and $\neg$ behavior. Cogprints.org.

Piccini, G., \& Scarantino, A. (2011). Information processing, computation, and cognition. Journal of Biological Physics, 37, 1-38. DOI10.1007/s10867-010-9195-3 Prinz, J. J. (2006). Is the mind really modular? Retrieved December 24, 2017 from http://www.subcortex.com/PrinzModularity.

Statistics Finland. (1989). Classification of socio-economic groups. Retrieved January 10, 2019 from http://www.stat.fi/meta/luokitukset/sosioekon_asema/001-1989/index_en.html 